\title{
Estado nutricional y hábitos alimenticios en niños de un colegio público de Valledupar
}

\author{
Bacterióloga. Magister en Ciencias Básicas Biomédicas. Docente Universidad de Santander sede Valledupar. \\ Bacterióloga. Especialista en Laboratorio de Hematología. Docente Universidad de Santander sede Valledupar. \\ Bacterióloga. Magister en Salud Pública. Docente Universidad de Santander sede Valledupar. \\ Médico. Especialista en Epidemiología. Secretaría Local de Salud de Valledupar. \\ Correo electrónico: mariaisabel2805@yahoo.es
}

María Isabel Mosquera Heredia ${ }^{1}$, Mabely Juliana Mosquera Heredia ${ }^{2}$, Lina María De Armas Daza ${ }^{3}$, Yeli Yelitza Brito Redondo ${ }^{4}$

Fecha de Recepción: 14/12/2015

Fecha de Evaluación: 21/2/2015

Fecha de Solicitud de Correcciones: 11/3/2016

Fecha de Aceptación: 11/3/2016

\section{Resumen:}

Introducción: La desnutrición es una de las principales causas de deficiencias cognitivas en los niños lo que puede afectar su desarrollo motriz y rendimiento escolar; además de que conlleva a un mayor riesgo de enfermedades y genera grandes costos en el sistema de salud.

Objetivo: Determinar el estado nutricional según antropometría y perfil de hierro y su asociación a hábitos alimenticios en una población de escolares de 9-11 años de edad.

Método: Estudio descriptivo-transversal en 155 escolares. El estado nutricional se valoró mediante medidas antropométricas y pruebas del laboratorio para valorar el estado del metabolismo del hierro. Se aplicó el cuestionario de frecuencia de consumo utilizado en ENSIN-2010.

Resultados: El 7,1\% de los niños presentó delgadez, el 17,4\% riesgo de delgadez, el 18,7\% sobrepeso, y el 7,1\% obesidad. Con respecto al indicador Talla para la edad, el 1,3\% de la población resultó con talla baja, y el 11,6\% con riesgo de talla baja para la edad.

Según la valoración del metabolismo del hierro, el 7,1\%, 5,8\% y 3,9\% fueron clasificados en los estadios 1,2 y 3 respectivamente.

El bajo consumo de frutas se asoció con riesgo de talla baja, y el bajo consumo de morcilla y vísceras con alteraciones en el metabolismo del hierro. Por otro lado, se observó que el alto consumo de arroz y pastas influye positivamente en los indicadores de IMC y Talla/E. Conclusiones: Se requieren acciones que corrijan hábitos alimenticios inadecuados en los niños para prevenir bajo rendimiento escolar y enfermedades crónicas en la adultez.

Palabras Clave: estado nutricional, hábitos alimenticios, escolares.
Nutritional status and eating habits of children in a Public school in Valledupar

Abstract

Introduction: Malnutrition is a major cause of cognitive deficits in children which can affect motor development and school performance; besides leading to an increased risk of diseases and generates large costs on the health system. Objetive: To determine nutritional status by anthropometry and iron profile and its association with eating habits in a population of schoolchildren 9-11 years old.

Methods: descriptive cross-sectional study of 155 schoolchildren. Nutritional status was assessed by anthropometric measurements and laboratory tests to assess the status of iron metabolism. The food frequency questionnaire used in 2010 was applied ENSIN.

Results: $7.1 \%$ of the children had thinness, the risk of thinness $17.4 \%, 18.7 \%$ overweight and $7.1 \%$ obese. With regard to the size indicator for age, $1.3 \%$ of the population was stunted, and $11.6 \%$ at risk of low height for age.

According to the assessment of iron metabolism, 7.1\%, 5.8\% and 3.9\% were classified in stages 1, 2 and 3 respectively.

The low consumption of fruit was associated with risk of stunting and low consumption of sausage and viscera with alterations in iron metabolism. On the other hand, we observed that the high consumption of rice and pasta positively influences BMI indicators and Size / E. Conclusions: actions to correct inadequate food habits in children to prevent underachievement and chronic diseases in adulthood are required.

Key Words: nutritional status, eating habits, schoolchildren.

\section{Introducción}

El estado nutricional de los seres humanos es el resultado del balance entre ingesta y necesidades de calorías y nutrientes. Este balance repercutirá en el grado de bienestar del individuo, en el cual influyen la alimentación, el entorno socio-económico y cultural y otros factores medioambientales. Cuando este balance se altera ya sea por la falta o el exceso del consumo de energía y nutrientes, en un lapso de tiempo determinado, se expresa con bajo peso y pobre desarrollo o con sobrepeso u obesidad; esto aumenta las tasas de morbimortalidad a corto plazo debilitando el sistema inmunológico del paciente y a largo plazo aumentando el desarrollo de enfermedades crónicas en la adultez y el riesgo cardiovascular[1-3].

Una de las formas más simples para diagnosticar la malnutrición es la utilización de variables antropométricas, como el peso y la talla las cuales se construyen índices antropométricos, que pueden ser analizados en forma conjunta o separada a fin de evaluar el estado nutricional de un individuo o población [4]. Se ha discutido ampliamente que la talla puede ser particularmente útil como indicador de las condiciones socioeconómicas en los países en desarrollo, debido a que las poblaciones de niños y adolescentes que están pobremente alimentados y son susceptibles a infecciones repetitivas raramente crecen de forma adecuada [5], es decir, la longitud refleja mas los efectos acumulativos de las circunstancias desfavorables durante el período de crecimiento. Como indicador del estado nutricional, el peso corporal proporciona una mejor idea de los efectos a corto plazo y de los cambios en los componentes corporales [6]. En cuanto al indicador peso para la talla, es la relación que existe entre el peso de un individuo con respecto a su talla, es más utilizado en niños mayores de dos años, pero tiene el inconveniente que no permite identificar niños desnutridos crónicos adaptados[6].

El encarecimiento de los alimentos ha incrementado el número de personas abocadas a una situación de hambre; con lo que la cifra de población desnutrida en el mundo en 2.007 se elevó a 923 millones de individuos, según datos de la Organización de Naciones Unidas para la Agricultura y la Alimentación (FAO), quien ve alejarse irremediablemente la posibilidad de alcanzar uno de los objetivos de desarrollo del milenio que propone "Erradicar la pobreza extrema y el hambre"[7 
Por su parte, el Fondo de la naciones unidas para la infancia UNICEF, en su informe del "Estado Mundial de la Infancia 2007", revela que uno de cada cuatro niños (aproximadamente 146 millones), lo cual representa el 27\% de los menores de cinco años, tienen peso inferior al normal[8].

En contraste, en América Latina y el Caribe se han logrado reducir las tasas de peso inferior al normal en los niños a un promedio de 3,8\% entre los años 1994 y 2.004[9]. Sin embargo, la infancia en esta región sufre aún serios problemas nutricionales como resultado de la existencia de graves disparidades y de una delicada crisis socioeconómica[9]. En Colombia particularmente, más de 500.000 niños, el 13\% de la población infantil, sufre de desnutrición crónica[10], lo cual muestra una relación positiva, bastante marcada, con el nivel socioeconómico especialmente en las zonas urbanas[11].

De acuerdo con los resultados de la encuesta nacional de desnutrición ENSIN del Instituto Colombiano de Bienestar Familiar[10], en la región Caribe (en la cual se encuentra situado el departamento del Cesar y su capital Valledupar), el 14,5\% de su población presenta desnutrición crónica por encima del promedio nacional $(13,2 \%)$

Por otra parte, el consumo inadecuado de nutrientes tanto por exceso como por déficit, puede estar asociado con subconsumo de algunos micronutrientes como el hierro, el zinc, la vitamina A, entre otros. Estas deficiencias de micronutrientes, conocidas como hambre oculta, representan la forma de desnutrición mas generalizada en el mundo, tanto en países desarrollados como en países en vías de desarrollo [2, 12-13]

Los problemas de nutrición a largo plazo en américa Latina y el Caribe según la UNICEF son el retraso en el crecimiento y la anemia debido a la carencia de hierro[9]. Esta última afecta a cerca del $25 \%$ de la población mundial y durante el embarazo se asocia al nacimiento de bebés con bajo peso, partos prematuros, mortalidad materna y mortalidad fetal. La deficiencia de hierro es una de las principales causas de deficiencias cognitivas en los niños lo que puede afectar posteriormente su desarrollo motriz y su rendimiento escolar; además de que también altera el sistema inmunológico[14].

La anemia por deficiencia de hierro perjudica también la actividad económica de los individuos, las familias y las economías nacionales. El Comité Permanente de Nutrición de las Naciones Unidas ha calculado, por ejemplo, que los costos económicos de la anemia en Bangladesh ascienden al $7,9 \%$ de su producto interno bruto (PIB) [15].

Esta situación en general llama la atención de investigadores y entes gubernamentales, pues la malnutrición especialmente en la edad infantil se constituye en un obstáculo que le impide a la sociedad desarrollar todo su potencial, teniendo en cuenta que afecta la capacidad intelectual y cognitiva del niño, disminuyendo su rendimiento escolar y el aprendizaje de habilidades para la vida. Lo cual limita la capacidad del niño de convertirse en un adulto que pueda contribuir, a través de su evolución humana y profesional, al progreso de su comunidad y de su país" [14]

Esta investigación tuvo como objetivo determinar el estado nutricional por antropometría y perfil de hierro en un grupo de escolares de nueve a once años de un colegio público de Valledupar y su relación con los hábitos alimentarios.

\section{Materiales y métodos}

\section{Población y muestra:}

La población la conformaron los 571 escolares con edades entre los nueve y once años de edad del colegio Nacional Loperena de Valledupar (Sedes Loperena principal, Santo Domingo y Vicente Roig y Villalba). En esta investigación participaron 155 escolares. Para calcular la muestra se tuvo en cuenta un $95 \%$ del nivel de confianza, un error de muestreo del $5 \%$ y un porcentaje de ocurrencia del $10 \%$; según la Encuesta Nacional de Situación Nutricional ENSIN, el porcentaje de ocurrencia de desnutrición por indicadores antropométricos para niños de 0 a 9 años es del $9 \%$ y para aquellos de 10 a 17 años es del $10 \%[10]$; en este estudio escogimos el del $10 \%$ puesto que con este porcentaje el tamaño muestral es mayor. Con estos datos, el tamaño de la muestra se estimó en 111 niños, que para efectos de contemplar perdidas sin afectar el tamaño muestral, este valor fue ajustado a 155 individuos, los cuales fueron seleccionados al azar mediante muestreo aleatorio simple.

Los criterios de inclusión fueron: estudiante matriculado para el periodo 2014 en el colegio Nacional Loperena (En cualquiera de sus tres sedes) con edades comprendidas entre los 9 y los 11 años, con ayuno previo y con consentimiento de los padres o acudientes, y asentimiento del niño. Los criterios de exclusión fueron: niños que no quisieron participar en el estudio, no tener autorización de padres o acudientes, no estar escolarizado en el colegio o ser estudiante temporal, no tener la edad requerida, no estar en ayuno.

\section{Variables estudiadas: \\ Hábitos alimenticios:}

Las madres o acudientes responsables de la alimentación de los niños fueron citados al colegio para aplicarles un instrumento de frecuencia de consumo de alimentos. A aquellos que no pudieron asistir, el instrumento se les aplicó vía telefónica. Este cuestionario fue el mismo utilizado en ENSIN-2010. Se tuvieron diez opciones de respuesta: tres o más veces por día, dos veces por día, una vez al día, cinco a seis veces por semana, tres a cuatro veces por semana, una vez por semana, dos a tres veces por mes, una vez al mes, < una vez al mes, nunca.

Las posibles limitaciones del método de frecuencia de consumo tales como una lista incompleta de todos los alimentos y errores en la estimación de la frecuencia y de las porciones; se controlaron utilizando un cuestionario validado por ENSIN-2010, en el cual se agruparon alimentos con características nutricionales comunes con base en el perfil de nutrientes de los alimentos colombianos, las problemáticas de mayor interés en salud pública y las recomendaciones de expertos[10]; además con este cuestionario no se buscaba obtener información de aporte de nutrientes ni del tamaño de las porciones de los alimentos.

\section{Estado nutricional:}

\section{Clasificación según pruebas antropométricas:}

Los niños se pesaron con ropa ligera sin zapatos y después de vaciar la vejiga. El peso se registró en kilogramos con un decimal. La estatura se midió con tallímetro de madera y se registró en centímetros con un decimal, siguiendo los parámetros establecidos en el protocolo de toma de medias antropométricas del Ministerio de Protección Social, 2.010. El índice de masa corporal se calculó como kg/m2. Para disminuir el sesgo de error, se realizó la toma de cada uno de los datos antropométricos tres veces y se obtuvo el promedio de los tres datos.

Para la toma de medidas antropométricas se utilizó como técnica normativa, lo estipulado en la resolución 2121 de 2.010 del ministerio 
de protección social. En el cuadro uno se describen los puntos de corte para cada indicador:

Cuadro 1. Puntos de Cohorte OMS, para la población Colombiana. Resolución 2121 de 2.010

\begin{tabular}{ccl}
\hline Indicador & $\begin{array}{c}\text { Punto de corte } \\
(\text { DS })\end{array}$ & \multicolumn{1}{c}{ Interpretación } \\
\hline & $<-2$ & $\begin{array}{l}\text { Talla baja para la edad o retraso } \\
\text { en talla } \\
\text { Riesgo de talla baja }\end{array}$ \\
& $>-2$ a $<-1$ & Talla adecuada para la edad \\
& $>-1$ & Delgadez \\
IMC / E & $>-2$ & Riesgo para delgadez \\
& $>-1$ a 1 & Adecuado para la edad \\
& $>\mathbf{1}$ a 2 & Sobrepeso \\
& $>\mathbf{2}$ & Obesidad \\
\hline
\end{tabular}

T: talla, E: edad, IMC: Índice de Masa Corporal.

\section{Anemia por deficiencia de hierro:}

Para establecer la prevalencia de anemia por deficiencia de hierro en la población estudiada, se realizaron las siguientes pruebas del laboratorio clínico: Hemoglobina $(\mathrm{Hb})$, índices eritrocitarios: ancho de distribución eritrocitaria (RDW), volumen corpuscular medio (VCM), hemoglobina corpuscular media (HCM), hierro sérico (HS), ferritina sérica (FS) y capacidad de la fijación total del hierro (CFTH).

El porcentaje de saturación de la transferrina $(\% \mathrm{ST})$ se calculó así= $\% \mathrm{ST}=\mathrm{HS} \times 100 / \mathrm{CFTH}$

Con estas pruebas se pudo clasificar a los pacientes según la alteración en el metabolismo del hierro de la siguiente manera:

- Paciente normal: Hb, índices eritrocitarios, HS, FS, CFTH y \%ST normales

- Estadío 1: Reserva deficiente de hierro: FS disminuida, RDW disminuido, todo lo demás normal.

- Estadío 2: Eritropoyesis deficiente en hierro: RDW, VCM, HCM, FS, HS y \%ST disminuidos; CFTH aumentada y Hb normal.

- Estadío 3: Anemia por deficiencia de hierro: RDW, VCM, HCM, FS, HS y \%ST disminuidos; CFTH aumentada y $\mathrm{Hb}$ disminuida.

\section{Análisis estadístico:}

No se excluyó ningún registro. Se analizó la totalidad de la muestra. Se calcularon los promedios de las variables estudiadas según edad y sexo. Se efectuaron comparaciones de medias con la prueba de ANOVA. Se calcularon las prevalencias para cada estado nutricional y del metabolismo del hierro según edad y sexo y se establecieron las diferencias estadísticas entre las prevalencias mediante la prueba de chi cuadrado. El valor de significancia estadística se estableció a partir de $\mathrm{p}<0,05$. Para ello se utilizó el programa estadístico SPSS versión 20.0 .

\section{Resultados:}

Se estudiaron 155 escolares con edades comprendidas entre los nueve y once años (la totalidad de la muestra fue analizada. No hubo registros excluidos), de los cuales el $62,6 \%$ pertenecía al género femenino y el $37,4 \%$ al género masculino. En la tabla dos se exponen las medidas antropométricas y las medidas del laboratorio clínico de la población estudiada según edad y sexo.
Adicionalmente, se estudiaron algunas variables que podrían influir en el estado nutricional del niño, entre ellas se encuentran: Nivel educativo de la madre, familiares con los que vive el niño, y edad y ocupación del acudiente. Con relación con esto, se pudo observar que las madres del 9,0\% de los niños analizados no tenían ningún tipo de estudios o escasamente terminaron la primaria, el 12,2\% de los menores convivían con sus abuelos; y con respecto a los acudientes, predominaron aquellos con edades entre los 30 y 40 años y los que se dedicaban al hogar con porcentajes del $55,5 \%$ y $27,1 \%$ respectivamente.

Estado nutricional de los escolares mediante pruebas antropométricas

En el cuadro dos se exponen los resultados obtenidos de las medidas antropométricas en la muestra analizada. Se puede observar que el peso y la talla incrementaron significativamente con la edad, sin mayor diferencia entre varones y mujeres.

De acuerdo a lo estipulado en la resolución 2121 de 2.010 del Ministerio de la Protección Social, se encontró que el 1,3\%de la población estudiada resultó con talla baja para la edad, y el 11,6\% con riesgo de talla baja. Con respecto al indicador de IMC, se estableció que el $7,1 \%$ de los niños presentó delgadez, el 17,4\% riesgo de delgadez, el $18,7 \%$ sobrepeso y el $7,1 \%$ obesidad. En la tabla tres se muestra la clasificación del estado nutricional de los escolares de acuerdo a las medidas antropométricas según edad y sexo.

Se destaca que no se encontraron varones con talla baja, sin embargo, ellos presentan la mayor prevalencia de "Riesgo de talla baja", el cual también fue más frecuente en los escolares de 11 años de edad ( $\mathrm{p}>$ $0,05)$.

La prevalencia de delgadez fue mayor en las niñas que en los niños y en el grupo de menor edad. Por su parte el sobrepeso y la obesidad fueron más frecuentes en los varones y en los niños de 10 a 11 años de edad respectivamente. En ninguno de los casos se observó diferencia estadística.

$\mathrm{Al}$ analizar la malnutrición con las variables socioeconómicas se encontró que todos los niños con talla baja tenían madre sin estudios académicos y que el 18,2\% de los niños con delgadez y el 11,2\% de los escolares con riesgo de talla baja, cuentan con madres que escasamente terminaron sus estudios de primaria. En contraste, cerca del $90 \%$ de los niños con talla adecuada tienen madres bachilleres, técnicos o profesionales $(\mathrm{p}>0,05)$. Llama la atención que más de la mitad de los niños con exceso de peso tienen madres con estudios superiores $(55,2 \%$ de los escolares con sobrepeso y el $63,6 \%$ de los obesos) $(\mathrm{p}>0,05)$. 
Cuadro No. 2 Medidas antropométricas y características de laboratorio de los escolares estudiados según edad y sexo.

\begin{tabular}{lccccccc}
\hline Medidas Antropométricas & \multicolumn{10}{c}{ Edad (Años) } & \multicolumn{4}{c}{ Sexo } \\
& $\mathbf{9}$ & $\mathbf{1 0}$ & $\mathbf{1 1}$ & Valor de $\mathbf{p}$ & Femenino & Masculino & Valor de p \\
Peso $(\mathbf{K g})$ & $30,0 \pm 7,3$ & $34,5 \pm 8,2$ & $38,6 \pm 7,9$ & 0,002 & $34,09 \pm 8,4$ & $34,1 \pm 8,5$ & 0,955 \\
Talla $(\mathbf{c m})$ & $134 \pm 0,6$ & $140 \pm 0,5$ & $145 \pm 0,6$ & 0,003 & $140 \pm 0,8$ & $139 \pm 0,7$ & 0,306 \\
IMC $\left(\mathbf{K g} / \mathbf{m}^{2}\right)$ & $16,5 \pm 2,9$ & $17,3 \pm 3,5$ & $18,1 \pm 2,7$ & 0,029 & $17,1 \pm 3,0$ & $17,2 \pm 3,1$ & 0,494 \\
\hline
\end{tabular}

\begin{tabular}{|c|c|c|c|c|c|c|c|c|c|c|c|}
\hline \multicolumn{12}{|l|}{ Pruebas hematológicas } \\
\hline & \multicolumn{5}{|c|}{ Edad (Años) } & \multirow[t]{2}{*}{ Valor de $p$} & \multicolumn{3}{|c|}{ Sexo } & \multirow[t]{2}{*}{ Valor de p } & \multirow[t]{2}{*}{ IBR } \\
\hline & \multicolumn{3}{|c|}{10} & \multicolumn{2}{|l|}{11} & & \multicolumn{2}{|c|}{ Femenino } & Masculino & & \\
\hline Hemoglobina (gr/dL) & $13,0 \pm 0,7$ & \multicolumn{2}{|c|}{$12,9 \pm 0,7$} & \multicolumn{2}{|c|}{$13,1 \pm 0,6$} & 0,393 & \multicolumn{2}{|c|}{$13,0 \pm 0,7$} & $13,0 \pm 0,6$ & 0,731 & $11,5-14 \mathrm{~g} / \mathrm{dL}$ \\
\hline $\operatorname{ADE}(\%)$ & $13,2 \pm 1,6$ & \multicolumn{2}{|c|}{$13,4 \pm 1,7$} & \multicolumn{2}{|c|}{$13,6 \pm 1,5$} & 0,532 & \multicolumn{2}{|c|}{$13,6 \pm 1,4$} & $13,1 \pm 1,8$ & 0,071 & $11,0-15,5 \%$ \\
\hline VCM (fL) & $78,9 \pm 5,4$ & \multicolumn{2}{|c|}{$79,3 \pm 5,6$} & \multicolumn{2}{|c|}{$79,6 \pm 5,1$} & 0,138 & \multicolumn{2}{|c|}{$80,3 \pm 4,9$} & $78,5 \pm 5,3$ & 0,043 & 75-100 fL \\
\hline HCM (pg) & $26,2 \pm 2,1$ & \multicolumn{2}{|c|}{$26,8 \pm 2,2$} & \multicolumn{2}{|c|}{$27,5 \pm 1,6$} & 0,008 & \multicolumn{2}{|c|}{$26,9 \pm 2,0$} & $26,5 \pm 2,1$ & 0,256 & $37-32 \mathrm{pg}$ \\
\hline \multicolumn{12}{|l|}{ Pruebas bioquímicas } \\
\hline & \multicolumn{6}{|c|}{ Edad (Años) } & Valor de $p$ & \multicolumn{2}{|c|}{ Sexo } & Valor de p & IBR \\
\hline & \multicolumn{2}{|l|}{9} & \multicolumn{2}{|c|}{10} & & 11 & & Femenino & Masculino & & \\
\hline Hierro sérico (ug/dL) & $66,8 \pm 2$ & 26,5 & 69,8 & $\pm 28,9$ & 77 & $\pm 24,8$ & 0,103 & $70,2 \pm 25,9$ & $72,7 \pm 28,9$ & 0,59 & $60-120 \mathrm{ug} / \mathrm{dL}$ \\
\hline Ferritina sérica (ug/L) & $49,3 \pm 1$ & 19,5 & 49,8 & $\pm 21,1$ & 51 , & $\pm 21,9$ & 0,841 & $50,8 \pm 20,3$ & $42,9 \pm 21,4$ & 0,645 & $30-140 \mathrm{ug} / \mathrm{L}$ \\
\hline Transferrina (\%) & $35,5 \pm 1$ & 12,2 & 36,0 & $\pm 13,3$ & & $2 \pm 9,4$ & 0,093 & $38,0 \pm 10,9$ & $35,7 \pm 13,4$ & 0,263 & $15-50 \%$ \\
\hline CFTH (ug/dL) & $204 \pm$ & & 214 & \pm 95 & & $9 \pm 64$ & 0,692 & $193 \pm 72$ & $206 \pm 84$ & 0,013 & $100-400 \mathrm{ug} / \mathrm{dL}$ \\
\hline
\end{tabular}

Valor de p calculado con ANOVA. ADE= Ancho de Distribución Eritrocitaria, VCM= Volumen Corpuscular Medio, HCM= Hemoglobina

Corpuscular Media, CFTH= Capacidad de Fijación Total del Hierro. Todos los valores son expresados como promedio y Desviación Estándar DS. IBR= Intervalo Biológico de Referencia.

\section{Anemia por deficiencia de hierro}

Los resultados de los parámetros hematológicos y bioquímicos que permiten valorar el estado del metabolismo del hierro se muestran en el cuadro tres.

Cuadro No. 3. Estado Nutricional de los escolares de acuerdo a sus medidas antropométricas según edad y sexo.

\begin{tabular}{|c|c|c|c|c|c|}
\hline \multicolumn{6}{|c|}{ IMC (Indice de Masa Corporal) } \\
\hline & \multicolumn{3}{|c|}{ Edad (Años) } & \multicolumn{2}{|c|}{ Sexo } \\
\hline & 9 & 10 & 11 & Femenino & Masculino \\
\hline Delgadez & $10,30 \%$ & $6,10 \%$ & $4,20 \%$ & $7,20 \%$ & $6,90 \%$ \\
\hline Riesgo de delgadez & $15,50 \%$ & $24,50 \%$ & $12,50 \%$ & $18,60 \%$ & $15,50 \%$ \\
\hline IMC adecuado & $56,90 \%$ & $38,80 \%$ & $52,10 \%$ & $50,40 \%$ & $48,30 \%$ \\
\hline Sobrepeso & $8,60 \%$ & $20,40 \%$ & $29,20 \%$ & $18,60 \%$ & $19,00 \%$ \\
\hline Obesidad & $8,60 \%$ & $10,20 \%$ & $2,10 \%$ & $5,20 \%$ & $10,30 \%$ \\
\hline Valor de $\mathrm{p}$ & & 0,078 & & & \\
\hline \multicolumn{6}{|l|}{ Talla para la edad } \\
\hline & \multicolumn{3}{|c|}{ Edad (Años) } & \multicolumn{2}{|c|}{ Sexo } \\
\hline & 9 & 10 & 11 & Femenino & Masculino \\
\hline Talla baja & $1,70 \%$ & $0,00 \%$ & $2,10 \%$ & $2,10 \%$ & $0,00 \%$ \\
\hline Riesgo de talla baja & $8,60 \%$ & $10,20 \%$ & $16,70 \%$ & $8,20 \%$ & $17,20 \%$ \\
\hline Talla adecuada & $89,70 \%$ & $89,80 \%$ & $81,20 \%$ & $89,70 \%$ & $82,80 \%$ \\
\hline Valor de p & \multicolumn{3}{|c|}{0,59} & \multicolumn{2}{|c|}{0,141} \\
\hline
\end{tabular}

Se observa que el Volumen Corpuscular Medio (VCM) fue significativamente mayor en el sexo femenino y la Capacidad de la Fijación total del hierro lo fue en los varones. Así mismo la Hemoglobina Corpuscular Media (HCM) fue menor en el grupo de niños con nueve años de edad (p $<0,05)$.

Según el estado del metabolismo del hierro se pudo establecer que el 3,9\% de los escolares tenían anemia por deficiencia de hierro, el 5,8\% eritropoyesis deficiente en hierro y el 7,1\% reserva deficiente en hierro. 
En el cuadro cuatro se expone la caracterización de la población estudiada de acuerdo al estado del metabolismo del hierro según edad y sexo. Se destaca que la anemia fue significativamente mayor en el sexo femenino y en los niños más pequeños, aunque no hubo diferencia estadística según edad.

Cuadro $\mathrm{N}^{\circ}$ 4. Estado del metabolismo del hierro según edad y sexo.

\begin{tabular}{lccccc}
\hline $\begin{array}{c}\text { Estado del } \\
\text { Metabolismo } \\
\text { del Hierro }\end{array}$ & $\mathbf{9}$ & $\mathbf{1 0}$ & $\mathbf{1 1}$ & Femenino & Masculino \\
\hline Normal & $82,8 \%$ & $81,6 \%$ & $85,4 \%$ & $88,7 \%$ & $74,1 \%$ \\
Estadío 1 & $6,9 \%$ & $4,1 \%$ & $10,4 \%$ & $5,2 \%$ & $10,3 \%$ \\
Estadío 2 & $5,2 \%$ & $10,2 \%$ & $2,1 \%$ & $2,1 \%$ & $12,1 \%$ \\
Estadío 3 & $5,2 \%$ & $4,1 \%$ & $2,1 \%$ & $4,1 \%$ & $3,4 \%$ \\
Valor de p & & 0,557 & & \multicolumn{2}{c}{0,035} \\
\hline
\end{tabular}

Valor de p calculado con Chi cuadrado.

Hábitos alimenticios y su relación con el estado nutricional de los niños

Para analizar los hábitos alimenticios se realizaron agrupaciones de los alimentos prioritarios de la siguiente manera: lácteos, carnes y/o huevos, frutas, verduras y hortalizas, y vísceras y morcilla. En la figura No. uno se expone el consumo diario de estos grupos de alimentos.

Figura No. 1. Proporciones de las frecuencias diarias de consumo por grupos de alimentos prioritarios

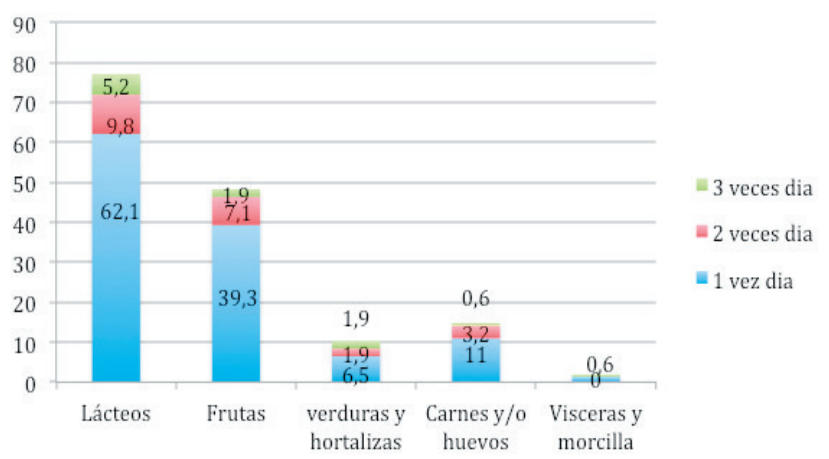

En el caso de los lácteos se incluyen alimentos como la leche líquida y en polvo, y sus derivados tales como queso, yogurt y kumis. En general el $100 \%$ de los padres manifestaron que sus hijos los consumen diariamente, y como se observa en la figura uno, más de la mitad de ellos lo hace una sola vez al día. Este grupo de alimentos es el más frecuentemente consumido por los niños estudiados.

Cerca del $49 \%$ de los estudiantes consumen frutas habitualmente. De ellos el $85 \%$ las consumen entera y el $97 \%$ en jugos.

Llamala atenciónelbajoconsumodecarnesy/ohuevosenestapoblación. En este grupo de alimentos se incluyeron las carnes rojas, el pollo /gallina, atún/sardina, pescados y mariscos y los huevos.

El grupo de alimento menos consumido lo conforman las vísceras y la morcilla seguido por las verduras y hortalizas. Más de la mitad de los niños consumen las vísceras, pero por lo general lo hacen 1 vez al mes. Menos del 1\% lo hace diariamente. En el caso de las verduras, solo el $10 \%$ de los niños las consumen diariamente, y de ellos la gran mayoría las prefieren crudas (97\%).
Respecto a la frecuencia de consumo de alimentos preparados e industrializados, se tiene que más del $60 \%$ de los niños consumen diariamente gaseosas, dulces y alimentos de paquetes; y el $70 \%$ y $50 \%$ de ellos consumen embutidos y comidas rápidas semanalmente (Figura número dos).

Figura No. 2. Frecuencia de consumo de alimentos preparados e industrializados

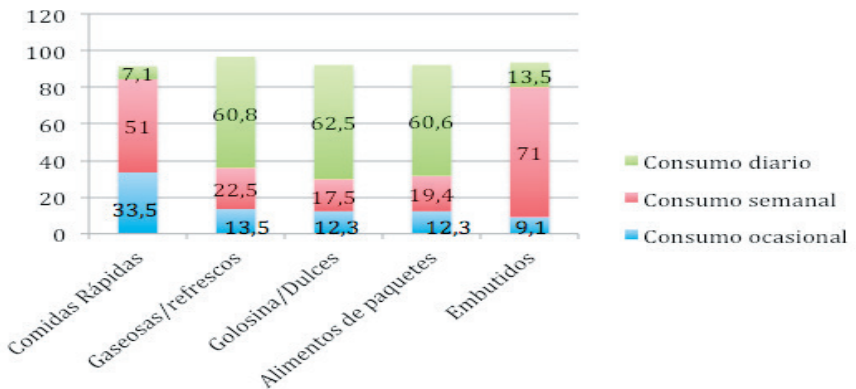

De acuerdo a la información presentada en la tabla cinco, se puede establecer que el hecho de que más de la mitad de los niños no consuman frutas diariamente, se asoció con riesgo de talla baja, y el bajo consumo de morcilla y vísceras con alteraciones en el metabolismo del hierro. Por otro lado, se observó que el alto consumo de arroz y pastas influye positivamente en los indicadores de IMC y Talla/E (Cuadro No. 5).

Cuadro No. 5. Asociación del estado nutricional con la frecuencia de consumo de alimentos

Alimentos prioritarios

\begin{tabular}{lccc}
\hline $\begin{array}{l}\text { Grupo de } \\
\text { alimentos }\end{array}$ & $\begin{array}{c}\text { Talla para la } \\
\text { edad } \\
\text { (Valor de p) }\end{array}$ & $\begin{array}{c}\text { IMC } \\
\text { (Valor } \\
\text { de } \mathbf{p} \text { ) }\end{array}$ & $\begin{array}{c}\text { Estado del metabolismo } \\
\text { del hierro }\end{array}$ \\
\hline $\begin{array}{l}\text { Lácteos } \\
\text { Frutas }\end{array}$ & 0,448 & 0,785 & 0,989 \\
$\begin{array}{l}\text { Verduras y } \\
\text { Hortalizas }\end{array}$ & 0,002 & 0,392 & 0,063 \\
$\begin{array}{l}\text { Carnes y/o } \\
\text { Huevos }\end{array}$ & 0,973 & 0,471 & 0,271 \\
$\begin{array}{l}\text { Visceras y } \\
\text { Morcilla }\end{array}$ & 0,142 & 0,106 & 0,506 \\
$\begin{array}{l}\text { Arroz y } \\
\text { Pastas }\end{array}$ & 0,003 & 0,037 & 0,026 \\
\hline
\end{tabular}

Alimentos preparados e industrializados

\begin{tabular}{lccc}
\hline $\begin{array}{l}\text { Grupo de } \\
\text { alimentos }\end{array}$ & $\begin{array}{c}\text { Talla para la } \\
\text { edad } \\
\text { (Valor de p) }\end{array}$ & $\begin{array}{c}\text { IMC } \\
\text { (Valor } \\
\text { de } \mathbf{p})\end{array}$ & $\begin{array}{c}\text { Estado del metabolismo } \\
\text { del hierro } \\
\text { (Valor de p) }\end{array}$ \\
$\begin{array}{l}\text { Embutidos } \\
\text { Comidas } \\
\text { rápidas }\end{array}$ & 0,132 & 0,96 & 0,967 \\
$\begin{array}{l}\text { Gaseosas/ } \\
\text { Refrescos }\end{array}$ & 0,777 & 0,271 & 0,023 \\
$\begin{array}{l}\text { Golosinas/ } \\
\text { Dulces } \\
\begin{array}{l}\text { Alimentos } \\
\text { de paquetes }\end{array}\end{array}$ & 0,918 & 0,503 & 0,356 \\
\hline
\end{tabular}

Valor de p calculado con Chi cuadrado 


\section{Discusión}

En la región de las Américas, los problemas de desnutrición coexisten cada vez más con problemas de sobrealimentación. En general la desnutrición afecta especialmente a los niños más pequeños y se manifiesta con retraso en el crecimiento (Talla baja para la edad) y anemia por deficiencia de hierro. Por su parte, el exceso de peso (Sobrepeso y obesidad) se está convirtiendo en un problema creciente que contribuye al desarrollo de enfermedades crónicas, como hipertensión, enfermedades cardiovasculares y diabetes mellitus tipo II en la adultez[16].

En la actualidad, Colombia y 188 países más, diseñan y ejecutan estrategias para alcanzar los denominados "Objetivos del Milenio" que pretenden mejorar la calidad de vida de la población. El primero de estos objetivos es "Erradicar la pobreza extrema y el hambre". Colombia para el logro de este, se propuso la meta de disminuir la prevalencia del indicador de "Retraso en la talla" a un $8 \%$ de niños y niñas con talla baja para la edad[17].

De acuerdo a la "Encuesta Nacional de la Situación Nutricional de Colombia” (ENSIN) realizada en el año 2.010, este indicador presentó una prevalencia del $9 \%$ y $10 \%$ en los niños de 5-9 años y de 10-17 años respectivamente[10].

En nuestro estudio, se reporta una prevalencia del 1,3\% de escolares de un colegio público de Valledupar con edades entre los nueve y once años. La diferencia entre estos resultados, se puede interpretar como producto de todas las acciones que en el país y específicamente en el departamento del Cesar se han implementado con el fin de alcanzar la meta nacional[18]. Debe tenerse en cuenta que este estudio se realizó en una muestra muy pequeña $(n=155)$ con lo cual no es posible concluir que en la ciudad de Valledupar o en el departamento del Cesar se halla superado la meta nacional del $8 \%$ de niños con baja talla, pero si se trata de una aproximación en cuanto al estado nutricional de los niños en edad escolar de la región.

En este sentido, la prevalencia del retraso de crecimiento en la población estudiada es baja, pero es importante continuar con el seguimiento de este indicador, pues como se expuso anteriormente, existen algunas condiciones socioeconómicas que están influyendo en la nutrición de los niños, como es el caso del nivel educativo de sus madres.

Otro indicador del estado nutricional de los menores es el Índice de Masa Corporal (IMC). Estudios anteriores dan cuenta del alarmante aumento del sobrepeso y la obesidad en la población infantil[19]. En esta oportunidad se encontró una prevalencia del $25,8 \%$ de exceso de peso ( $18,7 \%$ de sujetos con sobrepeso y $7,1 \%$ con obesidad).

Según cifras de la Organización Mundial de la Salud OMS, el aumento de este indicador en niños es constante a nivel mundial. En el África, la Américas y Asia; la prevalencia del sobrepeso aumentó entre uno y tres puntos porcentuales desde 1.990 hasta el año 2.013. Los datos que se tienen del continente Europeo muestran que este indicador pasó del 5,2\% al 6,3\% en solo cinco años[19].

En Colombia, se reportó en el 2.010 una prevalencia de sobrepeso del $18,9 \%$ en niños de 5 - 9 años y del $16,7 \%$ en la población de $10-17$ años; y de obesidad de 5,2\% y $3,4 \%$ respectivamente para estos grupos de edades[10].
Estudios más recientes realizados en otras regiones del país coinciden con el aumento del sobrepeso y la obesidad en los escolares, aunque reportan prevalencias mayores. En Cartagena por ejemplo, el exceso de peso tuvo un porcentaje del 47,6\% y en Medellín del 38,1\%[20-21]. En Valledupar se reportó en el año 2.011, una prevalencia del 6,6\% en niños escolares con las mismas edades de este estudio[22]. Esto demuestra como también en la ciudad, en solo tres años este indicador ha aumentado en la población infantil de acuerdo a la tendencia mundial.

Se pudo evidenciar también, que el sobrepeso fue aumentando a medida que aumentaba la edad. Es probable que los niños más pequeños reciban alimentación más equilibrada seleccionada por sus padres o tutores, mientras que los escolares mayores deciden su alimentación debido a la autonomía propia de su edad, prefiriendo alimentos que aportan más calorías de acuerdo con lo reportado por Piaggio en el año 2.011[23].

$\mathrm{Al}$ analizar el nivel de estudio de las madres con el estado nutricional de los niños, llama la atención que el exceso de peso fue común en los hijos de quienes tenían educación superior (Profesionales, Técnicas), lo cual va de la mano con la ocupación de la madre; pues una mujer preparada tendrá más deseo y facilidad para trabajar. Datar y colaboradores en el año 2.014, buscaron la relación entre las horas de trabajo de las madres con el aumento del Índice de Masa Corporal ICM de sus hijos. Sus resultados coincidieron con lo reportado anteriormente con respecto a la asociación positiva entre horas de trabajo materno y el exceso de peso en los niños. Adicionalmente, demostraron que los niños cuyas madres trabajan más, tienen comportamientos poco saludables como por ejemplo, la preferencia por alimentos procesados, o preparados fuera del hogar y el aumento del sedentarismo, predominando actividades como ver televisión y el uso de video juegos, por falta de tiempo para supervisión de los hijos o programación de actividades físicas extracurriculares[24].

Hasta este punto, se puede decir que el problema nutricional más frecuente en la población estudiada es el exceso de peso, hecho que merece atención, dado que la obesidad es un denominador común en todos los factores de riesgo cardiovascular, principalmente hipertensión arterial[25-26] y dismetabolia [27-29], además de ser un predictor de peso en la edad adulta.

Otra manera de conocer el estado nutricional de los individuos, es establecer si existe o no ganancia de micronutrientes.

La deficiencia de hierro en el organismo es la deficiencia alimentaria más frecuente en el mundo y conduce a la anemia ferropénica. Tiene una prevalencia del $24,8 \%$ en la población total, siendo los niños el grupo más vulnerable a esta afección luego de las mujeres en estado de embarazo. Se ha reportado que el $25,4 \%$ de los niños en edad escolar tiene deficiencia de hierro, lo que indica que cerca de 305 millones de sujetos de este grupo etario padecen de esta carencia nutricional[30].

En Colombia, el 7,5\% de los niños con edades entre 5 y 12 años padecen anemia por deficiencia de hierro, sin diferencias significativas por edad, sexo, etnia, nivel del SISBEN, área o región[10]; al igual que en este estudio, se reportó que la anemia disminuía a medida que aumentaba la edad. Los niños de 9 años presentaron un porcentaje de $5,2 \%$, los de 10 años 4,1\% y los de 11 años 2,1\%.

Las implicaciones de la anemia por deficiencia de hierro son muy variadas e incluyen efectos sobre la función y estructura gastrointestinal, inmunidad e infección, funciones neurológicas y 
físicas. A nivel del Sistema Nervioso Central SNC, el hierro está comprometido en muchos procesos que podrían afectar la conducta infantil y su desarrollo, con efectos a largo plazo sobre el rendimiento intelectual y físico de los niños[31-33].

En general, se requieren acciones que corrijan hábitos alimenticios inadecuados en los niños para prevenir bajo rendimiento escolar y enfermedades crónicas en la adultez.

\section{Conflictos de interés}

Los autores declaramos que no tenemos ningún conflicto de interés.

\section{Agradecimientos:}

Proyecto co-financiado por la Universidad de Santander y la Secretaría Local de Salud de Valledupar-Cesar. Convocatoria interna 2013, acta 041-13.

\section{Referencias}

1. Mengistu K, Alemu K, Destaw B. Prevalence of Malnutritionand Associated Factors Among Children Aged 6-59 Months at Hidabu Abote -District, North Shewa, Oromia Regional State. J Nutr Disorders Ther. 2013; T1: 001. 2. Hernández LO. Estado nutricional en adolescentes de una población suburbana de la ciudad de México. Rev Mex Pediatr 2003; 70(3):109-117. 3. Baker Jl, Olsen LW, Sorensen TI. Childhood body-mass index and the risk of coronary Herat disease in adulthood. N Engl J Med 2007; 357(23):2329-37.

4. Organización Mundial de la Salud. El estado físico: uso e interpretación de la antropometría. Informe de un comité de expertos de la OMS. serie de informes técnicos 854. Ginebra. 1995; 5-40.

5. Gopalan C. Heights of population and index of their nutrition and socioeconomic development. Bull Nutr Found. Ind; 1987; 8:1-5.

6. López Blanco M, Landaeta M. Manual de Crecimiento y Desarrollo. Sociedad Venezolana de Puericultura y Pediatría. Evaluación Nutricional Antropometrica .Fundacredesa. Capítulo III, 1991.

7. Organización de las naciones unidas para la agricultura y la alimentación. 2007. Tomado de: http://www.fao.org/news/story/ es/item/8882/icode/. Acceso el día 19 de mayo de 2015.

8. Estado mundial de la infancia. Fondo de la naciones unidad para la infancia UNICEF. New York. 2007.

9. Progreso para la Infancia. Un balance sobre la nutrición. Número 4, mayo de 2006. Tomado de: http://www.unicef.org/spanish/ progressforchildren/2006n4/index_latincaribbean.html. Acceso el día 19 de mayo de 2015.

10. Encuesta Nacional de la Situación Nutricional en Colombia. ENSIN . Instituto Colombiano de Bienestar Familiar 2010.

11. Flórez C, Nupia O. Desnutrición infantil en Colombia: inequidades y determinantes. Organización Panamericana de la Salud. Disponible en: http://www.bvsde.paho.org/texcom/nutricion/ D2001.pdf. Acceso el día 19 de mayo de 2015.

12. Solano L, Baron MA, Del Real S. Situación nutricional de preescolares, escolares, y adolescentes de Valencia, Carabobo, Venezuela. An Venez Nutr 2005; 18(1):72-76.

13. Landaeta M, García MN, Bosh V. Principales deficiencias de micronutrientes en Venezuela. Rev Esp Nutr Comunitaria 2003; 9(3):117-127.
14. La desnutrición infantil: Causas, consecuencias y estrategias para su prevención y tratamiento. UNICEF España. 2011.

15. Mason, J.; Rivers, J., y Helwig, C., "Recent Trends in Malnutrition in Developing Regions: Vitamin A deficiency, anemia, iodine deficiency, and child underweight", Food and Nutrition Bulletin, vol. 26, 2005, pp. 28-34.

16. Organización Panamericana de la Salud OPS. La salud en las Américas. Vol I. 194-196. Washington D.C: OPS.2002

17. Consejo Nacional de Política Económica y Social. Metas y estrategias de Colombia para el logro de los objetivos de desarrollo del milenio - 2015. Bogotá D.C. 2005. Departamento Nacional de Planeación, 1-69.

18. Cesar, informe sobre el estado de avance de los objetivos de desarrollo del milenio, estado de avance 2011. Recuperado el día 1 del mes de Septiembre del año 2014 de URL: http://www.pnud. org.co/2012/odm2012/odm_cesar.pdf

19. OMS. Global Health Observatory Data Repository. Joint Child Malnutrition estimates (UNICEF-WHO-WB). Global and regional trends by WHO regions, 1990-2003.

20. López, R., González, V. \& González, Y. Estado nutricional, sedentarismo y características familiares en escolarizados de seis a diez años de edad. Revista ciencias biomédicas, 2014; 5(1), 71-78.

21. Taborda P, Pérez M, Berbesi D. Funcionalidad familiar, seguridad alimentaria y estado nutricional en niños del Programa Departamental de Complementacion Alimentaria de Antioquia. Rev CES Med 2011:25(1):6-19.

22. Mosquera, M.I., Ospino, L., Mosquera, M.J., \& Ramírez, M. Factores de riesgo cardiovascular en niños de 8-11 años de cinco escuelas públicas de Valledupar- Cesar-Colombia. Revista Médica de Risaralda, 2011; 17 (1). 13-21.

23. Piaggio L, Concilio C, Rolón M, Macedra G, Dupraz S. Alimentación Infantil en el ámbito escolar: entre patios aulas y comedores. Rev Salud Colectiva. 2011:7(2):15-17.

24. Datar A, Nicosia N, Shier V. Maternal work and children's diet, activity, and obesity. 2014. Social Science \& Medicine, pg 196-204.

25. Freedman DS, Dietz WH, Srinivasan SR, Berenson GS. The relation of overweight to cardiovascular risk factors among children and adolescents: the Bogalusa Heart Study. Pediatrics 1999;103(6 pt1):1175-82.

26. Sorof JM, Lai D, Turner J, Poffenbarger T, Portman RJ. Overweight, ethnicity, and the prevalence of hypertension in school-aged children. Pediatrics 2004;113(3 pt1):475-82.

27. Hirschler V, Delfino A, Clemente G, Aranda C y cols. ¿Es la circunferencia de cintura un componente del síndrome metabólico en la infancia? Arch Argent Pediatr 2005;103(1):7-13.

28. Maffeis C, Corciulo N, Livieri C, Rabbone I, et al. Waist circumference as a predictor of cardiovascular and metabolic risk factors in obese girls. Eur J Clin Nutr 2003;57(4):566-72.

29. Korsten-Reck U, Kromeyer-Hauschild K, Baunstark M, Korsten K, Dickhuth H, Berg A. Frequency of secondary dyslipidemia in obese children. Vascular Health and Risk Management 2008; 4(5): 1089-94.

30. Benoist B et al., eds. Worldwide prevalence of anaemia 19932005. Base de datos mundial sobre la anemia de la OMS, Ginebra, Organización Mundial de la Salud, 2008.

31. Strauss R. Iron deficiency, infections and immune function: a reassessment. Am J Clin Nutr 1978; 31: 660-666.

32. Beard JL, Connor JR. Iron status and neural functioning. Ann Rev Nutr 2003; 23: 41-58.

33. Viteri F, Torun B. Anemia and physical work capacity. Clin Haematol 1974; 3: 609-626. 